\title{
L-Carnitine as an Adjuvant Treatment in Acute Organophosphorus Pesticides Poisoning: A Randomized Clinical Trial
}

\author{
Mona M. Ghonem ${ }^{1}$, Heba I. Lashin ${ }^{1}$, Aliaa A. Hodeib ${ }^{1}$, Nema A. Soliman²
}

\begin{abstract}
KEYWORDS

Organophosphorous, Pesticides,

Malondialdehyde, Reduced glutathione, Serum total antioxidant Capacity,

L-carnitine

Organophosphorus compounds are widely used pesticides. They are associated with a significant risk of acute intoxication. Oxidative stress is a contributing factor of acute organophosphorus poisoning morbidity and mortality. L-carnitine was found to have free-radical scavenging and antioxidant properties. Therefore, we aimed to evaluate the efficacy and safety of L-carnitine as an adjuvant in treatment of patients with acute organophosphorus poisoning. A randomized clinical trial was conducted on 30 patients suffering from acute organophosphorus poisoning admitted to Poison Control Center, Tanta University Emergency Hospital, Egypt, from April 2017 till January 2018. Patients were randomly divided into two equal groups. Group I received the standard treatment only and group II received the standard treatment plus L-carnitine in a dose of $1 \mathrm{gm} / 8$ hours IV.At time of admission, malondialdehyde, reduced glutathione, serum total antioxidant capacity and psudocholinesterase enzyme activity registered no significant difference between the two studied groups. After treatment, malondialdehyde, reduced glutathione and serum total antioxidant capacity showed significant improvement in group II. The mean value of atropine dose in group II $(5.6 \mathrm{mg})$ was significantly lower than group I $(10.9 \mathrm{mg})$. We concluded that the use of L-carnitine improved the antioxidant status and reduced total atropine dose required for treatment of patients with acute organophosphorus poisoning.
\end{abstract}

\section{Introduction}

Organophosphorus (OP) compounds are widely used pesticides because of their relatively low cost and rapid degradation (Coskun et al., 2015; Gündüz et al., 2015). However, they are associated with a significant risk of acute intoxication especially in developing countries owing to unrestricted sale, lack of protective equipment and poorly

\footnotetext{
${ }^{(1)}$ Forensic Medicine and Clinical Toxicology Department, Faculty of Medicine, Tanta University.

${ }^{(2)}$ Medical Biochemistry Department, Faculty of Medicine, Tanta University.
}

educated farmers (Kir et al., 2012; Saad et al., 2017).

In Egypt, acute OP poisoning is a toxicological problem of considerable concern. According to Ibrahim et al. (2011), insecticide intoxication represented $49 \%$ of the total number of chemical poisoning and OP insecticides accounted for $55 \%$ of insecticide poisoned cases. El-Maddah (2012) found that OP poisoning was the second most common incidence $(30.4 \%)$ of acute poisoning.

Manifestations of acute OP poisoning are mediated mainly through irreversible inhibition of cholinesterase enzyme with subsequent 
accumulation of acetylcholine and overstimulation of muscarinic and nicotinic receptors (Shah and Mundhra, 2016). Moreover, different studies showed that metabolism of OP compounds by cytochrome $\mathrm{P} 450 \mathrm{~s}$ is associated with production of reactive oxygen species making oxidative stress another factor contributing to acute OP poisoning morbidity and mortality (Ranjbar et al., 2005; Kumar et al., 2010; Hundekari et al., 2013; Mirakbari, 2015).

Oxidative stress markers can be used for evaluation and monitoring of $\mathrm{OP}$ poisoned patients (Lukaszewicz-Hussain, 2010). As lipid peroxidation is considered one of the molecular mechanisms of acute OP poisoning, so malondialdehyde (MDA) is a suitable marker to assess oxidative status in acute OP poisoning (Hundekari et al., 2011). Reduced glutathione (GSH) is a major antioxidant that protects cells against reactive oxygen species (Mercer et al., 2016). Measurement of total antioxidant capacity (TAC) is one of the common strategies to assess free radical/antioxidant equilibrium (Fraga et al., 2014).

Stabilization of the patients followed by administration of atropine and oximes and decontamination are considered the corner stone of acute OP poisoning treatment (Bajracharya et al., 2016). Beside standard treatment, antioxidant medications may have a promising role through counteracting increased reactive oxygen species and enhancement of antioxidant system (Žunec et al., 2014).

L-carnitine (LC); a non-protein amino acid; is synthesized in mammalian liver, kidney and brain (Cao et al., 2011). It was found to have free-radical scavenging and antioxidant properties. It is considered a safe antioxidant where mild nausea and vomiting are major side effects (Singh and Aslam, 1998). In addition, it is used in different pathological conditions associated with increased oxidative stress and can ameliorate oxidative damage in animal models (Calo et al., 2005). Therefore, this study aimed to evaluate efficacy and safety of LC as an adjuvant in treatment of acute OP intoxicated patients.

\section{Patients and Methods}

\section{Study Design and Population:}

An open-label, phase II trial was conducted on 30 patients suffering from acute OP poisoning admitted to Poison Control Center, Tanta University Emergency Hospital, Tanta, Egypt, from April 2017 till January 2018.

Diagnosis is based on history of exposure, availability of poison bottle or label, characteristic odor of gastric contents, typical clinical toxidrome of cholinergic and nicotinic manifestations following exposure to $\mathrm{OP}$ and reduced serum pseudocholinesterase (PChE) activity below reference range.

Poisoning severity was graded according to Minton and Murray (1988) and Bey et al.(2001) into mild (fatigue, headache, blurred vision, dizziness, nausea, vomiting, excessive sweating, salivation, abdominal pain and chest tightness), moderate (symptoms of mild poisoning plus muscular fasciculation, weakness, inability to walk, chest crepitations and miosis) and severe (symptoms of moderate poisoning plus unconsciousness, flaccid paralysis, respiratory distress, cyanosis and marked miosis with loss of pupil reflexes).

Inclusion criteria:All symptomatic patients aged 18 years or more with acute OP poisoning were included.

Exclusion criteria: Patients aged less than 18 years, pregnant and lactating females, patients with mixed intoxication and debilitated or cachectic patients were excluded. Patients with medical conditions that may induce 
oxidative stress as cardiovascular diseases, renal, hepatic failure or neurodegenerative disorders and those presented more than 12 hours after exposure to OP compounds or received medical treatment for OP poisoning before admission were also excluded.

\section{Methods}

Thirty patients were randomly divided into two equal groups (15 patients each) by using the sequentially numbered, opaque, sealed, envelopes method (Doig and Simpson, 2005). Group I (standard treatment group) received standard treatment only and group II (L-carnitine group) received standard treatment plus LC.

Patients of both groups received standard treatment as guided by attending physician according to Tanta Poison Control Center Protocol.This standard treatment included patient resuscitation (if indicated), dermal or gastric decontamination with administration of single dose activated charcoal $(1 \mathrm{gm} / \mathrm{kg})$ and antidotal therapy (atropine and oxime). Atropine was given as a bolus dose of 1 to 3 mg IV, repeated every 10 to 15 minutes till full atropanization, then maintained by continuous infusion of $10-20 \%$ of the loading dose every hour (Eddleston et al., 2008). Toxogonin ${ }^{\circledR}$ (1 ampoule contains $250 \mathrm{mg}$ of obidoxime chloride in $1 \mathrm{ml}$, produced by Merck, Darmstadt, Germany) was given when nicotinic manifestations appeared as a loading dose of $250 \mathrm{mg}$ bolus IV, followed by $750 \mathrm{mg}$ every 24 hours until at least 12 hours after stopping of atropine (Roberts and Aaron, 2007). Patients of group II received LC in a dose of $1 \mathrm{gm} / 8$ hours IV (Elabbassia et al., 2014). It was given till stopping of standard treatment. Patients were closely monitored to detect any adverse effects of LC. If any occurred, LC was immediately stopped and the patient was registed as having side effect of the drug.

For all patients, sociodemographic and toxicological data were collected, physical examination including measurement of vital signs was done and blood samples were obtained at time of admission and before administration of any treatment. In adults, the normal ranges of blood pressure, pulse, respiratory rate and temperature are 90-130/60$90 \mathrm{mmHg}, \quad 60-100$ beats/min., $16-24$ cycles/min. and $36.5-37.5^{\circ} \mathrm{C}$ respectively (Flomenbaum et al., 2006 and Middleton, 2008).

An arterial blood sample (one $\mathrm{ml}$ ) was obtained from each patient for blood gas analysis according to Kokholm (1990). A venous blood sample (five $\mathrm{ml}$ ) of each patient was kept into a clean dry centrifuge tube and left to stand for few hours before centrifugation to avoid hemolysis, then serum was separated. Routine investigations including liver enzymes according to Reitman and Frankel (1957), blood urea according to Fawcett and Scott (1960) and Chaney and Marbach (1962), serum creatinine according to Houot (1985) and serum electrolytes according to Woo (1999) were measured.

Serum PChE activity was measured using butyrylthiocholine substrate according to Blawen et al. (1983). Serum level of MDA, GSH and TAC were assayed according to Satoh (1978), Tietez (1969) and Koracevic et al. (2001) respectively. Each of PChE activity, MDA, GSH and TAC were measured twice; at admission and when standard treatment was stopped.

\section{Outcome measures:}

The primary outcome was mortality, whereas secondary outcomes included hospital stay, total doses of atropine and oxime 
received, occurance of atropine complications and the need of ICU admission and/or mechanical ventilation.

\section{Ethical consideration:}

The study was carried out after approval of the research ethics committee of Tanta Faculty of Medicine (Approval Number: 31451/03/17). A written informed consent was taken from each patient or his/her guardians (if the patient was unfit for giving consent) after receiving detailed information about the study. To conserve confidentiality, code number was given for each patient and data were analyzed anonymously. The disposal of blood collection syringes, tubes and body fluids (blood samples) was safely done to avoid any risk of environmental pollution.

\section{Statistical Analysis}

The collected data were organized and statistically analyzed using SPSS statistical software version 22 for windows. For quantitative data, the Shapiro-Wilk test for normality was performed. For data that were not normally distributed median and interquartile range (expressed as 25th-75th percentiles) were calculated and MannWhitney was used for comparison between groups. For normally distributed data, values were expressed as mean \pm standard deviation and independent samples $\mathrm{T}$ test was used for comparison between two groups while paired $t$ test was used to compare two related samples. For qualitative data, Pearson's Chi square, Fisher's exact, or Fisher-Freeman-Halton exact tests were used to examine association between two variables. Significance was adopted at $p<$ 0.05 for interpretation of results of tests (Dawson and Trapp, 2001).

\section{Results}

The present study was carried out on 30 patients with acute OP poisoning admitted to Tanta Poison Control Center. Patients were divided into two groups randomly. Sociodemographic and toxicological data of the studied patients were demonstrated in table (1). Age of participants showed mean values 32.8 and 33.3 years in group I and II respectively. Male patients represented $60 \%$ of group I and $66.7 \%$ of group II. There was no significant difference between the two groups regarding sociodemographic and toxicological data at time of admission. 
Table (1): Evaluation of sociodemographic and toxicological data of the studied acute organophosphrus poisoned patients $(n=30)$.

\begin{tabular}{|c|c|c|c|c|c|c|}
\hline \multirow{2}{*}{\multicolumn{2}{|c|}{ Iriables }} & \multicolumn{3}{|c|}{ Groups } & \multicolumn{2}{|c|}{ Tests of significance } \\
\hline & & \multirow{2}{*}{$\begin{array}{c}\begin{array}{c}\text { Total } \\
(\mathbf{n}=\mathbf{3 0})\end{array} \\
33.1 \pm 11.8\end{array}$} & \multirow{2}{*}{$\begin{array}{c}\begin{array}{c}\text { Group I } \\
(\mathbf{n}=\mathbf{1 5})\end{array} \\
32.8 \pm 11.6\end{array}$} & \multirow{2}{*}{$\begin{array}{c}\begin{array}{c}\text { Group II } \\
(\mathbf{n}=\mathbf{1 5})\end{array} \\
33.3 \pm 12.3\end{array}$} & \multirow{2}{*}{$\begin{array}{c}\begin{array}{c}\text { Test } \\
\text { statistic }\end{array} \\
\mathrm{t}=-0.122\end{array}$} & \multirow{2}{*}{$\begin{array}{c}\mathbf{P} \\
0.904\end{array}$} \\
\hline Age (years) & Mean \pm SD & & & & & \\
\hline & & n (\%) & n (\%) & n (\%) & & \\
\hline \multirow{4}{*}{$\begin{array}{l}\text { Age groups } \\
\text { (years) }\end{array}$} & $18-30$ & $14(46.7 \%)$ & $7(46.7 \%)$ & $7(46.7 \%)$ & \multirow{4}{*}{$\begin{array}{l}\mathrm{X}_{\mathrm{FFH}}^{2} \\
=0.710\end{array}$} & \multirow{4}{*}{1.000} \\
\hline & $30-<40$ & $4(13.3 \%)$ & $2(13.3 \%)$ & $2(13.3 \%)$ & & \\
\hline & $40-<50$ & $9(30 \%)$ & $5(33.3 \%)$ & $4(26.7 \%)$ & & \\
\hline & $\geq 50$ & $3(10 \%)$ & $1(6.7 \%)$ & $2(13.3 \%)$ & & \\
\hline \multirow{2}{*}{ Sex } & Male & $19(63.3 \%)$ & $9(60 \%)$ & $10(66.7 \%)$ & \multirow{2}{*}{$\begin{array}{c}\mathrm{X}_{\mathrm{ChS}}^{2}= \\
0.144\end{array}$} & \multirow{2}{*}{0.705} \\
\hline & Female & $11(36.7 \%)$ & $6(40 \%)$ & $5(33.3 \%)$ & & \\
\hline \multirow{3}{*}{ Occupation } & Farmers & $18(60 \%)$ & $9(60 \%)$ & $9(60 \%)$ & \multirow{3}{*}{$\begin{array}{c}\mathrm{X}_{\mathrm{FFH}}^{2}= \\
2.880\end{array}$} & \multirow{3}{*}{0.208} \\
\hline & Housewives & $7(23.3 \%)$ & $2(13.3 \%)$ & $5(33.3 \%)$ & & \\
\hline & Students & $5(16.7 \%)$ & $4(26.7 \%)$ & $1(6.7 \%)$ & & \\
\hline \multirow{2}{*}{ Residence } & Rural & $24(80 \%)$ & $11(73.3 \%)$ & $13(86.7 \%)$ & \multirow{2}{*}{ FE } & \multirow{2}{*}{0.651} \\
\hline & Urban & $6(20 \%)$ & $4(26.7 \%)$ & $2(13.3 \%)$ & & \\
\hline \multirow{2}{*}{ Marital state } & Married & $21(70 \%)$ & $10(66.7 \%)$ & $11(73.3 \%)$ & \multirow{2}{*}{ FE } & \multirow{2}{*}{1.000} \\
\hline & Single & $9(30 \%)$ & $5(33.3 \%)$ & $4(26.7 \%)$ & & \\
\hline \multirow{2}{*}{ Route of exposure } & Oral & $14(46.7 \%)$ & $6(40 \%)$ & $8(53.3 \%)$ & \multirow{2}{*}{$\begin{array}{c}\mathrm{X}_{\mathrm{ChS}}^{2}= \\
0.536\end{array}$} & \multirow{2}{*}{0.464} \\
\hline & Combined & $16(53.3 \%)$ & $9(60 \%)$ & $7(46.7 \%)$ & & \\
\hline \multirow{2}{*}{ Mode of exposure } & Suicidal & $13(43.3 \%)$ & $6(40 \%)$ & $7(46.7 \%)$ & \multirow{2}{*}{$\begin{array}{c}\mathrm{X}_{\mathrm{ChS}}^{2}= \\
0.136\end{array}$} & \multirow{2}{*}{0.713} \\
\hline & Accidental & $17(56.7 \%)$ & $9(60 \%)$ & $8(53.3 \%)$ & & \\
\hline Delay time (hrs) & Mean \pm SD & $4.5 \pm 2.5$ & $4.7 \pm 2.4$ & $4.3 \pm 2.6$ & $\mathrm{t}=0.401$ & 0.691 \\
\hline
\end{tabular}

n: number; SD: standard deviation; $\mathrm{X}_{\mathrm{ChS}}^{2}$ : Pearson's Chi square test; $\mathrm{X}_{\mathrm{FFH}}^{2}$ : Fisher-Freeman-Halton Exact tests; FE:

Fisher's exact test; t: Independent samples t test, Group I: standard treatment group, Group II: L-carnitine group.

Regarding table (2), majority of the studied cases were evaluated as moderately intoxicated $(73.3 \%$ and $60 \%)$, while cases of mild intoxication constituted $(26.7 \%$ and $33.3 \%$ ) of group I and II respectively. Only one case was severely intoxicated in group II. No significant difference was observed between the studied groups regarding poisoning severity grading, vital signs and oxygen saturation on admission. 
Table (2): Assessment of vital signs, oxygen saturation and poisoning severity grading of the studied acute organophosphrus poisoned patients on admission $(n=30)$.

\begin{tabular}{|c|c|c|c|c|c|c|}
\hline \multirow{2}{*}{\multicolumn{2}{|c|}{ Variables }} & \multicolumn{3}{|c|}{ Groups } & \multicolumn{2}{|c|}{ Tests of significance } \\
\hline & & \multirow{2}{*}{\begin{tabular}{|c|}
$\begin{array}{c}\text { Total } \\
(\mathbf{n}=\mathbf{3 0})\end{array}$ \\
$\mathbf{n}(\mathbf{\%})$ \\
$16(53.3 \%)$
\end{tabular}} & \multirow{2}{*}{$\begin{array}{c}\text { Group I } \\
(\mathbf{n}=\mathbf{1 5}) \\
\mathbf{n}(\mathbf{\%}) \\
8(53.3 \%)\end{array}$} & \multirow{2}{*}{$\begin{array}{c}\text { Group II } \\
\begin{array}{c}(\mathbf{n}=\mathbf{1 5}) \\
\mathbf{n}(\%)\end{array} \\
8(53.3 \%)\end{array}$} & \multirow{4}{*}{$\begin{array}{c}\text { Test } \\
\text { statistic }\end{array}$} & \multirow{4}{*}{$\begin{array}{c}\mathbf{P} \\
1.000\end{array}$} \\
\hline \multirow{3}{*}{ Blood pressure } & Normal & & & & & \\
\hline & Hypotension & $10(33.3 \%)$ & $5(33.3 \%)$ & $5(33.3 \%)$ & & \\
\hline & Hypertension & $4(13.3 \%)$ & $2(13.3 \%)$ & $2(13.3 \%)$ & & \\
\hline \multirow{3}{*}{ Pulse } & Normal & $14(46.7 \%)$ & $6(40 \%)$ & $8(53.3 \%)$ & \multirow{3}{*}{$\begin{array}{c}\mathrm{X}_{\mathrm{FFH}}^{2}= \\
1.819\end{array}$} & \multirow{3}{*}{0.466} \\
\hline & Bradycardia & $15(50 \%)$ & $9(60 \%)$ & $6(40 \%)$ & & \\
\hline & Tachycardia & $1(3.3 \%)$ & $0(0 \%)$ & $1(6.7 \%)$ & & \\
\hline \multirow{3}{*}{ Temperature } & Normal & $15(50 \%)$ & $9(60 \%)$ & $6(40 \%)$ & \multirow{3}{*}{$\begin{array}{c}\mathrm{X}_{\mathrm{FFH}}^{2}= \\
1.343\end{array}$} & \multirow{3}{*}{0.573} \\
\hline & Decreased & $12(40 \%)$ & $5(33.3 \%)$ & $7(46.7 \%)$ & & \\
\hline & Increased & $3(10 \%)$ & $1(6.7 \%)$ & $2(13.3 \%)$ & & \\
\hline \multirow{3}{*}{ Respiration } & Normal & $14(46.7 \%)$ & $7(46.7 \%)$ & $7(46.7 \%)$ & \multirow{3}{*}{$\begin{array}{c}\mathrm{X}_{\mathrm{FFH}}^{2}= \\
0.144\end{array}$} & \multirow{3}{*}{1.000} \\
\hline & Bradypnea & $8(26.7 \%)$ & $4(26.7 \%)$ & $4(26.7 \%)$ & & \\
\hline & Tachypnea & $8(26.7 \%)$ & $4(26.7 \%)$ & $4(26.7 \%)$ & & \\
\hline \multirow{2}{*}{$\begin{array}{l}\text { Oxygen } \\
\text { saturation (\%) }\end{array}$} & $\begin{array}{l}\text { Minimum- } \\
\text { maximum }\end{array}$ & $88-98$ & $88-99$ & $88-98$ & \multirow[t]{2}{*}{$\mathrm{t}=1.346$} & \multirow{2}{*}{0.189} \\
\hline & Mean \pm SD & $94 \pm 3.3$ & $94.8 \pm 2.88$ & $93.2 \pm 3.59$ & & \\
\hline \multirow{3}{*}{$\begin{array}{l}\text { Poisoning } \\
\text { severity grading }\end{array}$} & Mild & $9(30 \%)$ & $4(26.7 \%)$ & $5(33.3 \%)$ & \multirow{3}{*}{$\begin{array}{c}\mathrm{X}_{\mathrm{FFH}}^{2} \\
=1.289\end{array}$} & \multirow{3}{*}{0.700} \\
\hline & Moderate & $\begin{array}{c}20 \\
(66.7 \%)\end{array}$ & $11(73.3 \%)$ & $9(60 \%)$ & & \\
\hline & Severe & $1(3.3 \%)$ & $0(0 \%)$ & $1(6.7 \%)$ & & \\
\hline
\end{tabular}

n: number; $\mathrm{X}_{\mathrm{FFH}}^{2}$ : Fisher-Freeman-Halton Exact tests; t: Independent samples $\mathrm{t}$ test, Group I: standard treatment group, Group II: L-carnitine group.

Table (3) showed that there was no statistical significant difference between group
I and II concerning routine laboratory investigations on admission. 
Table (3): Routine laboratory investigations of the studied acute organophosphrus poisoned patients on admission $(n=30)$.

\begin{tabular}{|c|c|c|c|c|c|c|c|c|}
\hline \multirow{3}{*}{ Variables } & \multicolumn{6}{|c|}{ Groups } & \multicolumn{2}{|c|}{$\begin{array}{c}\text { Independent } \\
\text { samples } \\
\text { t test }\end{array}$} \\
\hline & \multicolumn{2}{|c|}{$\begin{array}{c}\text { Total } \\
(n=30)\end{array}$} & \multicolumn{2}{|c|}{$\begin{array}{l}\text { Group I } \\
(n=15)\end{array}$} & \multicolumn{2}{|c|}{$\begin{array}{l}\text { Group II } \\
(\mathrm{n}=15)\end{array}$} & $\mathbf{t}$ & $\mathbf{P}$ \\
\hline & Range & Mean \pm SD & Range & $\operatorname{Mean} \pm$ SD & Range & $\operatorname{Mean} \pm$ SD & & \\
\hline $\operatorname{ALT}(\mathbf{U} / \mathbf{L})$ & $14-42$ & $22.2 \pm 6.8$ & $14-40$ & $21.9 \pm 6.5$ & $16-42$ & $22.5 \pm 7.4$ & -0.237 & 0.815 \\
\hline $\operatorname{AST}(\mathbf{U} / L)$ & $17-47$ & $29.4 \pm 9.7$ & $18-47$ & $28.8 \pm 10.3$ & $17-46$ & $22.9 \pm 9.5$ & -0.313 & 0.756 \\
\hline Urea (mg/dl) & $15-45$ & $27.6 \pm 7.5$ & $16-39$ & $26.9 \pm 6.6$ & $15-45$ & $28.3 \pm 8.6$ & -0.502 & 0.619 \\
\hline $\begin{array}{l}\text { Creatinine } \\
(\mathrm{mg} / \mathrm{dl})\end{array}$ & $0.6-1.2$ & $0.9 \pm 0.2$ & $0.7-1.2$ & $0.9 \pm 0.2$ & $0.6-1.2$ & $0.9 \pm .2$ & 0.096 & 0.925 \\
\hline pH & $7.36-7.503$ & $7.414 \pm .051$ & $7.36-7.503$ & $7.417 \pm .052$ & 7.34-7.502 & $7.411 \pm .053$ & 0.294 & 0.771 \\
\hline $\mathrm{PaCO}_{2}(\mathrm{mmHg})$ & $26.1-52.1$ & $41.3 \pm 7.2$ & $26.1-50.2$ & $39.2 \pm 7.0$ & $29.9-52.1$ & $43.3 \pm 7.0$ & -1.611 & 0.118 \\
\hline $\mathrm{HCO}_{3}(\mathrm{mmol} / \mathrm{l})$ & $20.4-33.2$ & $28 \pm 4$ & $21.7-33.2$ & $28 \pm 4$ & $20.4-32.9$ & $27 \pm 3$ & 0.712 & 0.482 \\
\hline $\mathrm{PaO}_{2}(\mathrm{mmHg})$ & $34.9-98.6$ & $77.3 \pm 15.6$ & $39.9-92.3$ & $76.0 \pm 15.4$ & $34.9-98.6$ & $78.6 \pm 16.1$ & -0.446 & 0.659 \\
\hline $\mathrm{Na}(\mathrm{mEq} / \mathrm{L})$ & $132.1-150.6$ & $140.9 \pm 5.5$ & $133.9-150.6$ & $141.4 \pm 6.0$ & 132.1-149.9 & $140.5 \pm 5.2$ & 0.431 & 0.670 \\
\hline $\mathbf{K}(\mathbf{m E q} / \mathbf{L})$ & $2.8-5.3$ & $3.9 \pm .6$ & $2.8-4.9$ & $3.8 \pm 0.6$ & $2.8-5.3$ & $4.0 \pm .7$ & -0.858 & 0.398 \\
\hline
\end{tabular}

SD: standard deviation; $\mathrm{n}$ : number; $\mathrm{t}$ : Independent samples $\mathrm{t}$ test; ALT: Alanine aminotransferase, AST, Aspartate aminotransferase. Group I: standard treatment group, Group II: L-carnitine group.

According to table (4), MDA, GSH, TAC and PChE activity registered no significant difference between the two studied groups on admission. After treatment, there was statistically significant difference between the two groups in MDA, GSH and TAC $(\mathrm{P}=$
$0.020,<0.001,<0.001)$ respectively. Whereas PChE activity did not show significant difference after treatment between the two groups $(\mathrm{p}=0.275)$. 
Table (4): Analysis of malondialdehyde, reduced glutathione, total antioxidant capacity and pseudocholineaterase enzyme activity in the studied acute organophosphorus poisoning patients on admission and after treatment $(n=30)$.

\begin{tabular}{|c|c|c|c|c|c|c|}
\hline \multirow[t]{2}{*}{ Variables } & \multirow[t]{2}{*}{ Time of analysis } & \multicolumn{3}{|c|}{ Groups } & \multicolumn{2}{|c|}{$\begin{array}{l}\text { Independent } \\
\text { samples t test } \\
\text { between two } \\
\text { groups }\end{array}$} \\
\hline & & $\begin{array}{c}\text { Total } \\
(n=30) \\
\text { Mean } \pm \text { SD }\end{array}$ & $\begin{array}{c}\text { Group I } \\
(n=15) \\
\text { Mean } \pm \text { SD }\end{array}$ & $\begin{array}{c}\text { Group II } \\
(n=15) \\
\text { Mean } \pm \text { SD }\end{array}$ & $\mathbf{t}$ & $\mathbf{P}$ \\
\hline \multirow{2}{*}{$\begin{array}{l}\text { MDA } \\
(\mathrm{nmol} / \mathrm{ml})\end{array}$} & On admission & $6.99 \pm 1.04$ & $6.97 \pm 1.01$ & $7.01 \pm 1.1$ & -0.107 & 0.915 \\
\hline & After treatment & $4.83 \pm 1.13$ & $5.3 \pm 0.86$ & $4.36 \pm 1.2$ & 2.477 & $0.020^{*}$ \\
\hline \multirow{2}{*}{ GSH (mg/dl) } & On admission & $1.02 \pm 0.38$ & $0.97 \pm 0.35$ & $1.07 \pm 0.42$ & -0.754 & 0.462 \\
\hline & After treatment & $1.75 \pm 0.69$ & $1.25 \pm 0.38$ & $2.24 \pm 0.57$ & -5.602 & $<0.001 *$ \\
\hline \multirow{2}{*}{ TAC(mmol/l) } & On admission & $0.80 \pm 0.24$ & $0.87 \pm 0.27$ & $0.74 \pm 0.21$ & 0.607 & 0.165 \\
\hline & After treatment & $1.67 \pm 0.73$ & $1.08 \pm 0.32$ & $2.25 \pm 0.5$ & -7.630 & $<0.001^{*}$ \\
\hline \multirow{2}{*}{$\begin{array}{l}\text { PChE activity } \\
\text { (U/L) }\end{array}$} & On admission & $\begin{array}{c}2781.8 \\
\pm 1353.2\end{array}$ & $\begin{array}{l}3134.3 \\
\pm 943.6\end{array}$ & $\begin{array}{c}2429.3 \\
\pm 1623.7\end{array}$ & 1.454 & 0.157 \\
\hline & After treatment & $\begin{array}{c}6112.5 \\
\pm 2329.1\end{array}$ & $\begin{array}{c}6584.3 \\
\pm 2091.6\end{array}$ & $\begin{array}{c}5640.8 \\
\pm 2526.9\end{array}$ & 1.114 & 0.275 \\
\hline
\end{tabular}

* significant; n: number; SD: standard deviation; t: Independent samples t test, Group I: standard treatment group, Group II: L-carnitine group.

The mean value of atropine dose in group II (5.6 mg) was significantly lower than in group I (10.9 mg). While, atropine complications appeared only in group I $(13.3 \%)$ with no significant difference between the two groups. The medians of toxogonin dose were 5 and 4 ampoules in group I and II respectively with no significant difference between the two studied groups. Mean values of hospital stay were 52.6 and 46.9 hours in group I and II respectively with no significant difference. Neither mortality nor the need for ICU admission and/or mechanical ventilation was recorded in both groups (Table 5). 
Table (5): Assessment of the outcome measures of the studied acute organophosphrus poisoned patients $(n=30)$.

\begin{tabular}{|c|c|c|c|c|c|c|}
\hline \multirow{2}{*}{\multicolumn{2}{|c|}{ Variables }} & \multicolumn{3}{|c|}{ Groups } & \multicolumn{2}{|c|}{ Tests of significance } \\
\hline & & \multirow{2}{*}{$\begin{array}{c}\begin{array}{c}\text { Total } \\
(\mathbf{n}=\mathbf{3 0})\end{array} \\
8.2 \pm 3.8\end{array}$} & \multirow{2}{*}{$\begin{array}{c}\begin{array}{c}\text { Group I } \\
(\mathbf{n}=\mathbf{1 5})\end{array} \\
10.9 \pm 3.4\end{array}$} & \multirow{2}{*}{$\begin{array}{c}\begin{array}{c}\text { Group II } \\
(\mathbf{n}=\mathbf{1 5})\end{array} \\
5.6 \pm 1.6\end{array}$} & \multirow{2}{*}{$\begin{array}{c}\begin{array}{c}\text { Test } \\
\text { statistic }\end{array} \\
\mathrm{t}=5.379\end{array}$} & \multirow{2}{*}{$\begin{array}{c}\mathbf{P} \\
<0.001^{*}\end{array}$} \\
\hline Atropine dose (mg) & Mean \pm SD & & & & & \\
\hline \multirow{2}{*}{ Toxogonin intake } & No (n, \%) & $12(40 \%)$ & $6(40 \%)$ & $6(40 \%)$ & \multirow{2}{*}{$\begin{array}{c}\mathrm{X}_{\mathrm{ChS}}^{2}= \\
0.000\end{array}$} & \multirow{2}{*}{1.000} \\
\hline & Yes $(n, \%)$ & $18(60 \%)$ & $9(60 \%)$ & $9(60 \%)$ & & \\
\hline \multirow{2}{*}{$\begin{array}{l}\text { Toxogonin dose in } \\
\text { ampoules } \\
\text { (Ampoule=250 } \mathrm{mg} \text { ) }\end{array}$} & Median (IQR) & $5(4-5)$ & $5(4-5)$ & $4(3-5)$ & \multirow{2}{*}{$\begin{array}{c}\mathrm{Z}_{\mathrm{MW}}=- \\
1.303\end{array}$} & \multirow{2}{*}{0.193} \\
\hline & Mean ranks & & 11.1 & 7.9 & & \\
\hline \multirow{2}{*}{$\begin{array}{l}\text { Atropine } \\
\text { complications }\end{array}$} & Present (n, \%) & $2(6.7 \%)$ & $2(13.3 \%)$ & $0(0 \%)$ & \multirow{2}{*}{$\mathrm{FE}$} & \multirow{2}{*}{0.483} \\
\hline & Absent (n, \%) & $28(93.3 \%)$ & $13(86.7 \%)$ & $15(100 \%)$ & & \\
\hline Hospital stay (hrs) & Mean \pm SD & $49.8 \pm 11.4$ & $52.6 \pm 11.7$ & $46.9 \pm 10.9$ & $\mathrm{t}=1.377$ & 0.180 \\
\hline
\end{tabular}

* : significant; n: number; SD: standard deviation; IQR: interquartile range; $\mathrm{Z}_{\mathrm{MW}}$ : Mann Whitney test; $\mathrm{X}_{\mathrm{ChS}}^{2}$ : Pearson's Chi square test; FE: Fisher's exact test; t: Independent samples $t$ test, Group I: standard treatment group, Group II: Lcarnitine Group.

\section{Discussion}

Acute OP poisoning is a major global clinical problem associated with high morbidity and mortality, especially in rural areas of developing countries (Eddleston et al., 2008; Dündar et al., 2015). Organophosphrus compounds irreversibly inhibit cholinesterase enzyme, causing excessive accumulation of acetylcholine with paralysis of cholinergic transmission in the central nervous system, autonomic ganglia, parasympathetic nerve endings, some sympathetic nerve endings and neuromuscular junction (Goel and Aggarwal, 2007).

This study was designed to evaluate the role of the antioxidant L-carnitine (LC) as an adjuvant therapy in acute OP poisoning. Findings of the current research regarding sociodemographic and toxicological characteristics and clinical data were more or less in accordance with previous studies performed in many Egyptian and international poisoning centers (Rehiman et al., 2008; Abd El AL et al., 2016; Kumar and Sahna, 2017).

Proper randomization using the sequentially numbered, opaque, sealed, envelopes method justifies lack of significant difference between the two groups regarding sociodemographic characteristics, toxicological and clinical data, vital signs, routine laboratory investigations, PChE activity and oxidative stress markers at the time of admission.

Herein, it was observed that MDA was significantly lower and each of GSH and TAC were significantly higher in group II versus group I after treatment. These findings were in accordance with previous experimental and 
clinical studies that showed that, use of antioxidants had a protective effect in acute OP poisoning (Shadnia et al., 2007; BalaliMood and Saber, 2012; El-Ebiary et al., 2016).

Organophosphorus compounds were found to generate excessive free radicals during their biotransformation, detoxification or even through mitochondrial impairment. This mitochondrial impairment can increase reactive oxygen species production with subsequent further mitochondrial damage generating a vicious circle (Abdel-Salam et al., 2016). Therefore, oxidative stress occurs as a result of imbalance between pro-oxidants and the antioxidant capacity with redox circuit disruption (Antonijevic et al., 2018).

In light of this data, it is expected to find improvement of oxidative stress parameters in group II patients who received LC which is a free radical scavenger. L-carnitine regulates enzymes activity involved in defense against oxidative damage and protects antioxidant enzymes; glutathione peroxidase, catalase and superoxide dismutase from oxidative damage (Uchenduet al., 2012; Mohammadi et al., 2018).

L-carnitine can facilitate $\beta$-oxidation of long-chain fatty acids, participate in branched chain amino acids metabolism and stabilize cellular membranes. Moreover, it can increase antioxidant enzymes activities in human (Lee et al., 2014). Furthermore, LC could improve antioxidant status and free radicals detoxification and provide strong antigenotoxic effect in OP intoxicated rats (Shokrzadeh et al., 2013; Shadboorestan et al., 2015). Additionally, LC administration to healthy volunteers significantly increased the antioxidant enzymes activities (Cao et al., 2011).

Data obtained from the current study revealed lack of significant difference between the two groups regarding PChE activity after treatment. Oximes can restore cholinesterase activity by removing phosphate group of the inhibited enzymes (Goel et al., 2012). In the current study, all cases were treated within 12 hours after exposure that allowed early oxime administration before aging of the inhibited enzyme. This could explain lack of significant difference between the two groups regarding $\mathrm{PChE}$ activity after treatment.

In this study, the dose of atropine in group II was significantly lower than in group I. This finding is in match with Shadnia et al. (2011) and El-Ebiary et al. (2016) who found that the use of the antioxidant $\mathrm{N}$-acetylcysteine significantly lowered the required atropine dose in comparison with patients who did not receive it.

L-carnitine has compensated consumption of antioxidant agents which could not be replaced in a short time. This might be utilized to overcome damage produced by free radicals and depletion of TAC resulted from OP poisoning (Eddleston et al., 2008). This could explain LC ability to ameliorate oxidative stress effect with subsequent reduction of atropine dose.

This study showed non-significant difference between the two groups regarding atropine complications. However, $13.3 \%$ of group I had atropine complications. This can be explained by the ability of the LC to reduce the required atropine dose with subsequent low incidence of atropine complications.

There was no significant difference between the two groups regarding toxogonin dose. This could be explained according to the current study by absence of significant difference between the two groups regarding PChE activity after treatment. Similar data were obtained by Shadnia et al. (2011) who found that, antioxidants use in acute OP poisoned patients had no effect on toxogonin dose. 
Despite there was no significant difference between the two groups regarding duration of hospital stay, it was slightly shorter in group II in comparison with group I. Shadnia et al.(2011) and Motawei and Elbiomy (2017) found that, N-acetylcysteine significantly lowered hospital stay in acute OP poisoned patients. Generally, the presence of other diseases or development of complications and even medical facilities in each country could affect the duration of hospital stay after poisoning (El-Naggar et al., 2009; Baydin et al., 2014).

\section{Conclusion}

The results of the current study declared that, LC improved antioxidant capacity and lowered atropine dose with subsequent lower incidence of atropine complications without any serious side effects. Additionally, LC is available in IV form so it can be easily administered to comatose patients. Hence, it is concluded that, LC could be considered a promising adjuvant antioxidant therapy in acute OP intoxication.

\section{Acknowledgement}

The authors are greatly thankful to all patients included in the current study.

\section{Study Limitations}

The major limitation of this study was the small sample size. Subsequently, severe cases were limited. Therefore, further studies with larger sample size in different poisoning centers are suggested to confirm the results of the current study. However, to the author's best knowledge; this is the first clinical trial to assess LC as adjuvant therapy in treatment of acute OP toxicity in Egypt.

\section{References}

Abd El AL, A.H.; Fawzi, M.M.; ALKhafif, M.A. and El-Zemait, M.E. (2016): "Epidemiological study of organophosphorus compounds insecticides types related to acutely intoxicated patients presented to Poison Control Center (PCCASU)-Egypt". IOSR Journal of Environmental Science, Toxicology and Food Technology, 10(6):72-78.

Abdel-Salam, O.M.E.; Youness, E.R.; Mohammed, N.A.; et al. (2016): "Novel neuroprotective and hepatoprotective effects of citric acid in acute malathion intoxication". Asian Pacific Journal of Tropical Medicine, 9(12):1181-1194.

Antonijevic, E.; Kotur-Stevuljevic, J.; Musilek, K.; et al. (2018): "Effect of six oximes on acutely anticholinesterase inhibitor-induced oxidative stress in rat plasma and brain". Archives of Toxicology, 92(2):745-757.

Bajracharya, S.R.; Prasad, P.N. and Ghimire, R. (2016): "Management of organophosphorus poisoning". Journal of Nepal Health Research Council, 14(34):131-138.

Balali-Mood, M. and Saber, H.(2012):"Recent advances in the treatment of organophosphorous poisonings". Iran Journal of Medical Sciences, 37(2):74-91.

Baydin, A.; Erenler, A.K.; Yardan, T.; et al. (2014):"Acute organophosphate poisoning in adults: A 10-Year Analysis". Health MED Journal, 8(2):151-160.

Bey, T.A.; Sullivan, J.B. and Walter, F.G. (2001): Organophosphate and carbamate 
insecticides. In: Clinical Environmental Health and Toxic Exposures. Sullivan, J.B. and Krieger, G.R. (Eds.), $2^{\text {nd }}$ Edition. Lippincott Williams \& Wilkins, London, P.P.1046-1057.

Blawen, D.; Poppe, D.H. and Trischler, W.A. (1983): "Serum acetylcholinesterase assay". Journal of Clinical Chemistry and Clinical Biochemistry, 21(6):381-386.

Calo, L.A.; Pagnin, E.; Davis, P.A.; et al. (2005): "Antioxidant effect of Lcarnitine and its short chain esters relevance for the protection from oxidative stress related cardiovascular damage". International Journal of Cardiology, 107:54-60.

Cao, Y.; Qu, H. J.; Li, P.; et al. (2011): "Single dose administration of Lcarnitine improves antioxidant activities in healthy subjects". The Tohoku Journal of Experimental Medicine, 224(3):209-213.

Chaney, A.L. and Marbach, E.P. (1962):"Urea determination by phenol hypochlorite method using Berthelot reaction". Clinical Chemistry, 8:130132.

Coskun, R.; Gundogan, K.; Sezgin, G.C.; et al. (2015): "A retrospective review of intensive care management of organophosphate insecticide poisoning: Single center experience". Nigerian Journal of Clinical Practice, 18(5):644650 .

Dawson, B. and Trapp, R.G. (2001): Basic and clinical biostatistics, Lange Medical Book. $3^{\text {rd }}$ Edition. McGrow Hill Medical Publishing Division, New York, P.P. 161-218.

Doig, G.S. and Simpson, F. (2005): "Randomization and allocation concealment: A practical guide for researchers". Journal of Critical Care, 20:187-193.

Dündar, Z.D.; Köylü, R.; Ergin, M.; et al. (2015): "Prognostic value of red cell distribution width in patients with organophosphate poisoning". The Journal of Academic Emergency Medicine, 14(2):65-69.

Eddleston, M.; Buckley, N.A.; Eyer, P. and Dawson, A.H. (2008): "Management of acute organophosphorus pesticide poisoning". Lancet, 371(9612):597-607.

Elabbassia, W.; Chowdhuryb, M.A. and Fachtartza, A.A. (2014): "Severe reversible myocardial injury associated with aluminum phosphide toxicity: A case report and review of literature". Journal of the Saudi Heart Association, 26(4):216221.

El-Ebiary，A.A.; Elsharkawy，R.E.; Soliman, N.A.; Soliman M.A. and Hashem A.A. (2016): "N-acetylcysteine in acute organophosphorus pesticide poisoning: a randomized, clinical trial". Basic and Clinical Pharmacology and Toxicology, 119(2):222-227.

El-Maddah, E.I. (2012):"Pattern of acute poisoning in adult patients admitted to Tanta poison center-Egypt in 2011". Egyptian Journal of Forensic Sciences and Applied Toxicology, 12(1):63-77.

El-Naggar, A.R.; Abdalla, M.S.; El-Sebaey, A.S. and Badawy, S.M. (2009):"Clinical findings and cholinesterase levels in children of organophosphates and carbamate poisoning". European Journal of Pediatrics, 168(8):951-956.

Fawcett, I.K. and Scott, J.E. (1960): "Phenol hypochlorite method for urea determination". Journal of Clinical Pathology, 13:156-159. 
Flomenbaum, N.; Goldfrank, L.; Hoffman, R.; et al. (2006): Initial evaluation of the patient: Vital signs and toxic syndromes. In: Goldfrank's Toxicological Emergencies. Flomenbaum, N.; Goldfrank, L.; Hoffman, R.; et al. (Eds.), $\quad 8^{\text {th }}$ Edition. McGrw-Hill: London, P.P. 37- 42.

Fraga, C.G.; Oteiza, P.I. and Galleano, M. (2014): "In vitro measurements and interpretation of total antioxidant capacity". Biochimica et Biophysica Acta (BBA) - General Subjects, 1840 (2):931-934.

Goel, A. and Aggarwal, P. (2007):"Pesticide poisoning". National Medical Journal of India, 20(4):182-191.

Goel, P.; Gupta, N.; Singh, S.; et al. (2012): "Regeneration of red cell cholinesterase activity following pralidoxime (2-PAM) infusion in first $24 \mathrm{~h}$ in organophosphate poisoned patients". Indian Journal of Clinical Biochemistry, 27(1):34-39.

Gündüz, E.; Dursun, R.; Içer, M.; et al. (2015):"Factors affecting mortality in patients with organophosphate poisoning". The Journal of the Pakistan Medical Association, 65(9):967-972.

Houot, O. (1985): Kinetic determination of creatinine. In: Interpretation of Clinical Laboratory Tests. Siest, G. Henny, J. and Schiele, F. (Eds), $8^{\text {th }}$ Edition. Biochemical Publishers, P.P. 220-234.

Hundekari, A.I.; Suryakar, A.N. and Rathi, D.B. (2013): "Acute organophosphorus pesticide poisoning in North Karnataka, India: oxidative damage, haemoglobin level and total leukocyte". African Health Science, 13(1):129-136.

Hundekari, I.A.; Suryakar, A.N. and Rathi, D.B. (2011): "Oxidative stress and antioxidant status in acute organophosphorous pesticides poisoning cases of North Karnataka (India)". Journal of Environmental Health Research, 11(1):3944.

Ibrahim, M.A.; El Masry, M.K.; Moustafa, A.M.; Hagras, A.M. and Ali, N.M. (2011): "Comparison of the accuracy of two scoring systems in predicting the outcome of organophosphate intoxicated patients admitted to intensive care unit (ICU)". Egyptian Journal of Forensic Sciences, 1(1):41-47.

Kır, M.Z.; Öztürk, G.; Gürler, M.; et al. (2012): "Pesticide poisoning cases in Ankara and nearby cities in Turkey: An 11-year retrospective analysis". Journal of Forensic and Legal Medicine, 20(4): 274277.

Kokholm, G. (1990): "Simultaneous measurements of blood $\mathrm{pH}, \mathrm{PCO}_{2}, \mathrm{PO}_{2}$ and concentrations of hemoglobin and its derivatives-A multicentre study". Scandavian Journal of Clinical and Laboratory Investigation Supplements, 203:75-86.

Koracevic, D.; Koracevic, G. and Djordjevic, V. (2001): "Method for the measurement of antioxidant activity in human fluids". Journal of Clinical Pathology, 54(5):356361.

Kumar, R.C. and Sahna, E. (2017): "Correlation of serum pseudocholinesterase level and peradeniya organophosphorus poisoning scale with the severity and in hospital outcome of acute organophosphorus poisoning". International Journal of Contemporary Medical Research, 4(8):1702-1705.

Kumar, S.V.; Fareedullah, M.D.; Sudhakar, Y.; Venkateswarlu B. and Kumar E.A. (2010): "Current review on organo- 
phosphorus poisoning". Archives of Applied Science Research, 2(4):199215.

Lee, B.J.; Lin, J.S.; Lin, Y.C. and Lin, P.T. (2014): "Effects of L-carnitine supplementation on oxidative stress and antioxidant enzymes activities in patients with coronary artery disease: A randomized, placebo-controlled trial". Nutrition Journal, 13(1):79.

Lukaszewicz- Hussain, A. (2010): "Role of oxidative stress in organophosphate insecticide toxicity- Short review". Pesticide Biochemistry and Physiology, 98:145-150.

Mercer, S.W.; Fontaine, S.L.; Warr, C.G. and Burke, R. (2016): "Reduced glutathione biosynthesis in Drosophila melanogaster causes neuronal defects linked to copper deficiency". Journal of Neurochemistry, 137(3):360-370.

Middleton, $S$ (2008): Assessment and investigations of patients' problems. In: Physiotherapy for Respiratory and Cardiac Problems. Pryor, J.A. and Prasad, S.A. (Eds.), $4^{\text {th }}$ Edition. Churchill Livingston, Elsevier, P.P. 8-9.

Minton, N.A. and Murray, V.S. (1988): "A review of organophosphate poisoning". Medical Toxicology and Adverse Drug Experience, 3(5):350-375.

Mirakbari, S.M. (2015): "Hot charcoal vomitus in aluminum phosphide poisoning - A case report of internal thermal reaction in aluminum phosphide poisoning and review of literature". Indian Journal of Anesthesia, 59(7): 433-436.

Mohammadi, H.; Djalali, M.; Daneshpazhooh, M.; et al. (2018): "Effects of L-carnitine supplementation on biomarkers of oxidative stress, antioxidant capacity and lipid profile, in patients with pemphigus vulgaris: a randomized, double-blind, placebocontrolled trial". European Journal of Clinical Nutrition, 72(1):99-104.

Motawei, S.M. and Elbiomy, A.A. (2017): "Sodium bicarbonate and $\mathrm{N}$-acetyl cysteine in treatment of organophosphorus poisoning cases: A randomized controlled clinical trial". Toxicology, 3(123): 24762067.

Ranjbar, A.; Solhi, H.; Mashayekhi, F.J. and Abdollahi, M. (2005): "Oxidative stress in acute human poisoning with organophosphorus insecticides; A case control study". Environmental Toxicology and Pharmacology, 20(1):88-91.

Rehiman, S.; Lohani, S.P. and Bhattarai, M. (2008): "Correlation of serum cholinesterase level, clinical score at presentation and severity of organophosphorous poisoning". Journal of Nepal Medical Association, 47(170):47-52.

Reitman, S. and Frankel, S. (1957):"A colorimetric method for determination of serum glutamic oxalacetic and purivic transaminase". American Journal of Clinical Pathology, 28(1):58-63.

Roberts, D.M. and Aaron, C.K. (2007): "Managing acute organophosphorous pesticide poisoning". British Medical Journal, 334: 629-634.

Saad, S.; Ismail, M.; Hashish, R. K.; et al. (2017):"The effect of the management with fresh frozen plasma on the outcome of patients with acute organophosphate poisoning". International Journal of Surgery and Medicine, 3(2):70-77.

Satoh, K. (1978): "Serum lipid peroxide in cerebrovascular disorders determined by a new colorimetric method". Clinica Chimica Acta, 90(1):37-43. 
Shadboorestan, A.; Shokrzadeh, M.; Ahangar, N.; et al. (2015): "The chemoprotective effects of L-carnitine against genotoxicity induced by diazinon in rat blood lymphocyte". Toxicology and Industrial Health, 31(12):1334-1340.

Shadnia, S.; Ashrafivand, S.; Mostafalou, S. and Abdollahi, M. (2011):"Nacetylcysteine: a novel treatment for acute human organophosphate poisoning". International Journal of Pharmacology, 7(6):732-735.

Shadnia, S.; Dasgar, M.; Taghikhani, S.; et al. (2007): "Protective effects of $\alpha$ Tocopherol and N-acetyl-cysteine on diazinon-induced oxidative stress and acetylcholinesterase inhibition in rats". Toxicology Mechanisms and Methods, 17(2):109-115.

Shah, N.M. and Mundhra, S.H. (2016): "Clinical profile of organophosphate poisoning at a tertiary-care center". International Journal of Medical Science and Public Health, 5(8):1-5.

Shokrzadeh, M.; Ahangar, N.; Zargari, M.; et al. (2013): "Protective effect of Lcarnitine on level of malondialdehyde in diazinon-induced lipid peroxidation in rats". Journal of Mazandaran University of Medical Sciences, 22(97):198-206.
Singh, R.B. and Aslam, M. (1998): "Lcarnitine administration in coronary artery disease and cardiomyopathy". The Journal of the Association of Physicians of India, 46(9): 801-805.

Tietez, F. (1969): "Enzymatic method for quantitative determination of nanogram amounts of total and oxidized glutathione: Applications to mammalian blood and other tissues". Analytical Biochemistry, 27(3): 502-522.

Uchendu, C.; Ambali, S. F. and Ayo, J. O. (2012):"The organophosphate, chlorpyrifos, oxidative stress and the role of some antioxidants: A review". African Journal of Agricultural Research, 7 (18): 2720-2728.

Woo, O.F. (1999): Organophosphates and carbamates. In: Poisoning and Drug Overdose. Anderson, I.B. and Clark, R.F. (Eds.), $3^{\text {rd }}$ Edition. Appleton \& Lange, Stanford, P.P. 224-248.

Žunec, S.; Kopjar, N.; Želježić, D.; et al. (2014): "In vivo evaluation of cholinesterase activity, oxidative stress markers, cyto- and genotoxicity of K048 oxime - a promising antidote against organophosphate poisoning". Basic and Clinical Pharmacology and Toxicology, 114(4):344-351. 


\section{لـ كارنيتين كملاج مساعد في حالات التسمم الحاد بمبيدات الآفات العضوية الفوسفورية: تجربة سريرية عشوائية}

\section{منى محمد غنيم ، هبه ابراهيم لاشين ، علياي عبد الحكم هديب' ، نعمة على سليمان '}

'قسم الطب الثرعي و السموم الاكلينيكية كلية الطب - جامعة طنطا

بقسم الكيمياء الحيوية كلية الطب - جامعة طنطا

تعد مركبات الفوسفات العضوية واحدة من مبيدات الآفات المستخدمة على نطاق واسع. إلا أنها ترتبط بشكل كبير بحسات التسمم الحساد. ويعتبر الإجهاد التأكسدي أحد العو امل التي تساهم في الحسالات المرضية و الوفيات الناجمة عن التسمم الحاد بهذه المركبات. ونظر الخو اص عقار لـ كارنيتين الكاسحة للشوارد الحرة و المضادة للأكسدة فإن هدفنا من هذه الدراسـة هو تقييم فعاليـة وسـلامة لـ كارنيتين كعلاج مساعد في حالات التسمم الحاد بمركبات الفوسفات العضوية.وقد تم إجر اء تجربة سريرية عشو ائية على ثلاثين مريض يعانون من هن التسمح الحساد بمركبات الفوسفات العضوية و الذين تم إدخالهم إلى مركز مكافحة التسمم ، مستشفى الطوارئ

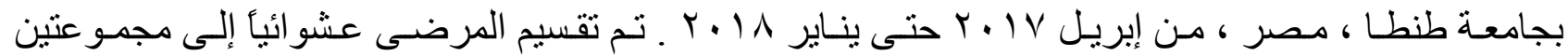
متساويتين. تلقت المجمو عة الأولى العلاج الأساسى فقط وتلقت المجمو عة الثانية العـلاج الأساسى بالإضـافة إلى الى لـ كارنيتين بجر عة ا جم / ^ ساعات وريديا. وقد أظهرت النتائج عدم وجود فرق ذى دلالة إحصائية بالنسبة لقياسـات المالوندهيـد ، الجلوتـاثيون المختـزل ، القـدرة التأكسدية الكليــة وانـزيم سـودو كـولين اسـتيريز بــين المجمـو عتين عنــ الـدخول. بينمـا أظهـرت الدر اسـة وجـود تحسن و اضـح بـين المجمـو عتين بالنـسبة لقياسـات

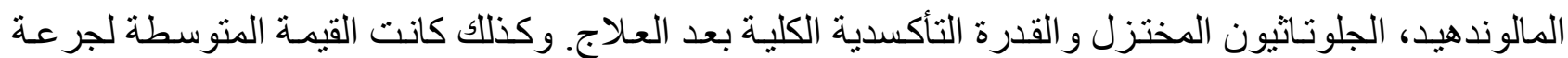

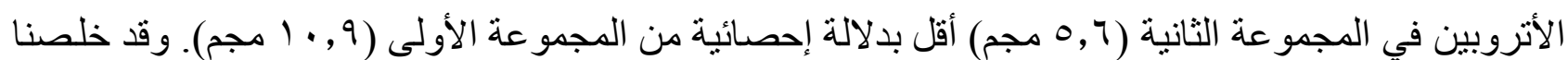

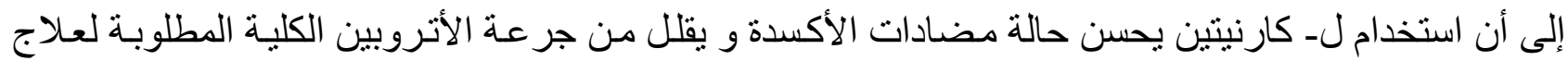
المرضى الذين يعانون من التسمم الحاد بمركبات الفوسفات العضوية. 